\title{
Trehalose conjugates of silybin as prodrugs for targeting toxic $A \beta$ aggregates
}

Sara García-Viñuales, ${ }^{1} \nabla$ Rashik Ahmed, ${ }^{2} \nabla$ Michele F. M. Sciacca ${ }^{1}$, Valeria Lanza ${ }^{1}$, Maria Laura Giuffrida ${ }^{1}$, Stefania Zimbone ${ }^{1}$, Valeria Romanucci, ${ }^{3}$ Armando Zarrelli, ${ }^{3}$ Corrado Bongiorno, ${ }^{4}$ Natalia Spinella, ${ }^{5}$ Clelia Galati, ${ }^{5}$ Giovanni Di Fabio, ${ }^{3 *}$ Giuseppe Melacini, ${ }^{2 *}$ and Danilo Milardi ${ }^{{ }^{*}}$

${ }^{1}$ Consiglio Nazionale delle Ricerche, Istituto di Cristallografia, Sede Secondaria di Catania, Via Paolo Gaifami 18, 95126 Catania, Italy; ${ }^{2}$ Departments of Chemistry and Chemical Biology \& Biochemistry and Biomedical Sciences, McMaster University, Hamilton ON, L8S 4M1, Canada; ${ }^{3}$ Department of Chemical Sciences, University of Naples Federico II, Via Cintia 4, I-80126 Napoli, Italy. ${ }^{4}$ Institute for Microelectronics and Microsystems, National Research Council, Stradale Primosole 50, 95121 Catania, Italy. ${ }^{5}$ STMicroelectronics, Stradale Primosole 50, 95121 Catania, Italy.

$\nabla_{\text {These authors equally contributed to this work }}$

\section{Electronic Supporting Information}


Table S1. Fitting parameters of $A \beta 40$ amyloid growth kinetics shown in Fig. 2.

\begin{tabular}{|c|c|c|c|c|}
\hline & $I_{\max }($ u.a. $)$ & $k\left(h^{-1}\right)$ & $t_{1 / 2}(h)$ & $t_{\text {lag }}(h)$ \\
\hline$A \beta$ & 49 & 0.3 & 26 & 20 \\
\hline$A \beta /$ Sil A-p-TH (1:0.4) & 45 & 0.7 & 38 & 32 \\
\hline A $\beta /$ Sil A-p-TH (1:1) & 40 & 0.5 & 42 & 36 \\
\hline$A \beta /$ Sil A-p-TH (1:5) & 43 & 0.5 & 44 & 40 \\
\hline$A \beta /$ Sil B-p-TH (1:0.4) & 41 & 0.6 & 29 & 24 \\
\hline Aß/Sil B-p-TH (1:1) & 37 & 0.6 & 36 & 33 \\
\hline$A \beta /$ Sil B-p-TH (1:5) & 44 & 0.7 & 50 & 38 \\
\hline$A \beta / T H(1: 5)$ & 49 & 0.32 & 27 & 23 \\
\hline
\end{tabular}



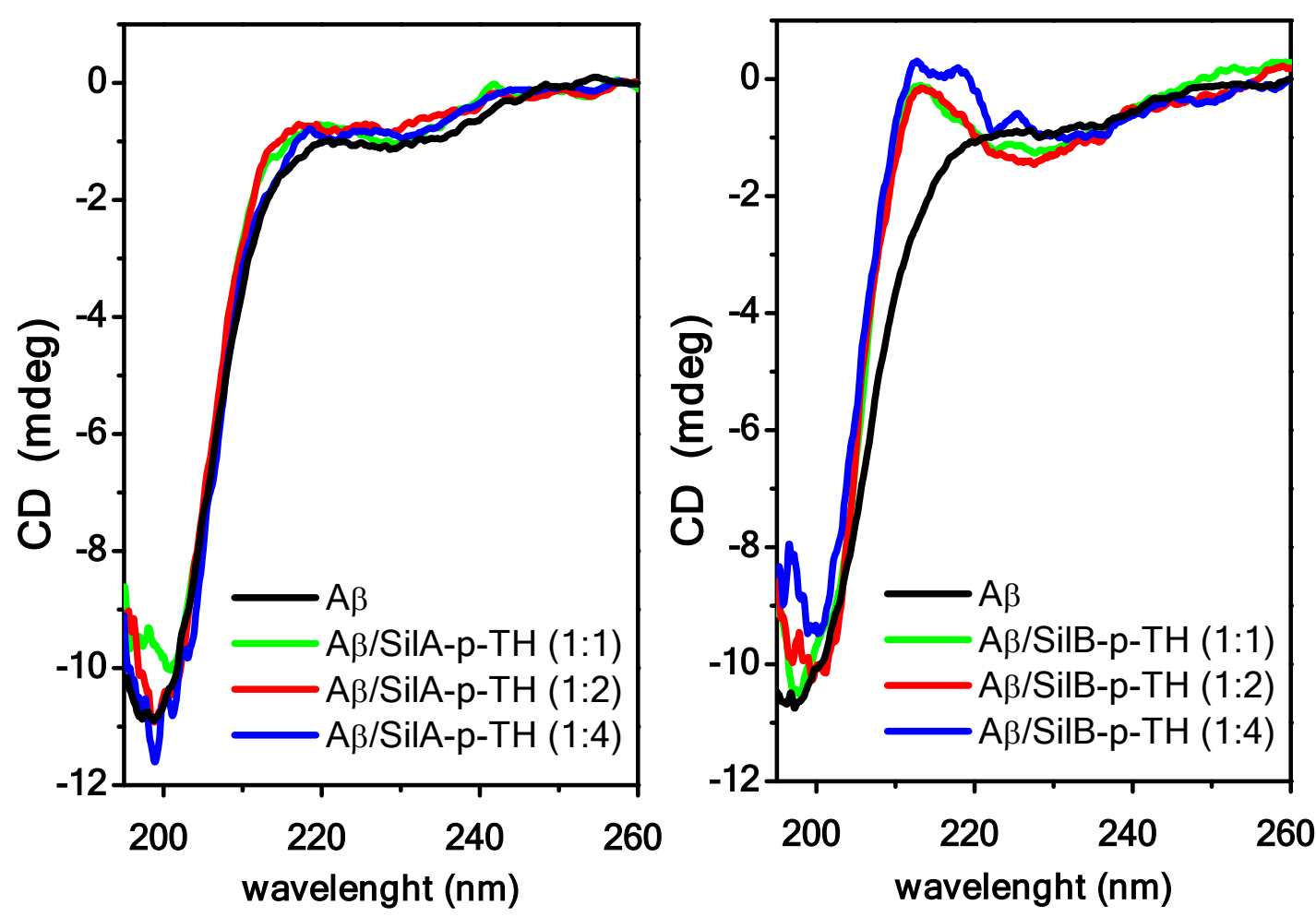

Figure S1. Left: CD spectra of $A \beta 40(2 \mu \mathrm{M})$ in the presence of Sil A-p-TH at peptide/ligand ratios 1:1, 1:2 and 1:4. Data were acquired at $4^{\circ} \mathrm{C}$ in $10 \mathrm{mM}$ phosphate buffer $\mathrm{pH}$ 7.4. Right: as left except for Sil B-p-TH.

\section{Effects of Sil A-p-TH and Sil B-p-TH on the conformational preferences of AB4O}

Based on ThT assays, we expect that the two silybin derivatives may interact with the amyloid peptide via different binding modes. To single out the ligand-induced $A \beta 40$ conformational changes for the two derivatives, we recorded Circular dichroism (CD) spectra of the peptide in the presence or absence of different concentrations of Sil A-p-TH and Sil B-p-TH. CD spectra of the pure ligands were recorded under the same experimental conditions and subtracted from the curves of the ligand/peptide mixtures. Unlike Sil A-p-TH which was essentially inactive, the difference spectra of $A \beta 40$ containing increasing amounts of Sil B-p-TH show a negative band at $\lambda=197 \mathrm{~nm}$ and a weak positive one at $214 \mathrm{~nm}$. Although it may be difficult to deconvolute these $C D$ spectra, as they likely reflect a complex mixture of slightly different conformations, this spectral signature is typical of a poly-L-proline II (PPII) helix structure (Fig. 4). PPII structure has been reported as indicative of the physiological/functional state of amyloidogenic proteins, since as it reduces the aggregation propensity of amyloidogenic peptides. ${ }^{1}$ Therefore, a shift in $A \beta$ conformation, stabilizing a PPII structure could be the cause of the antiaggregating effects observed in ThT 
experiments. Our CD results are in agreement with our ThT data and suggest that the major antiaggregating effect of Sil B-p-TH is related to its effect on the conformational preferences of $A \beta 40$. 


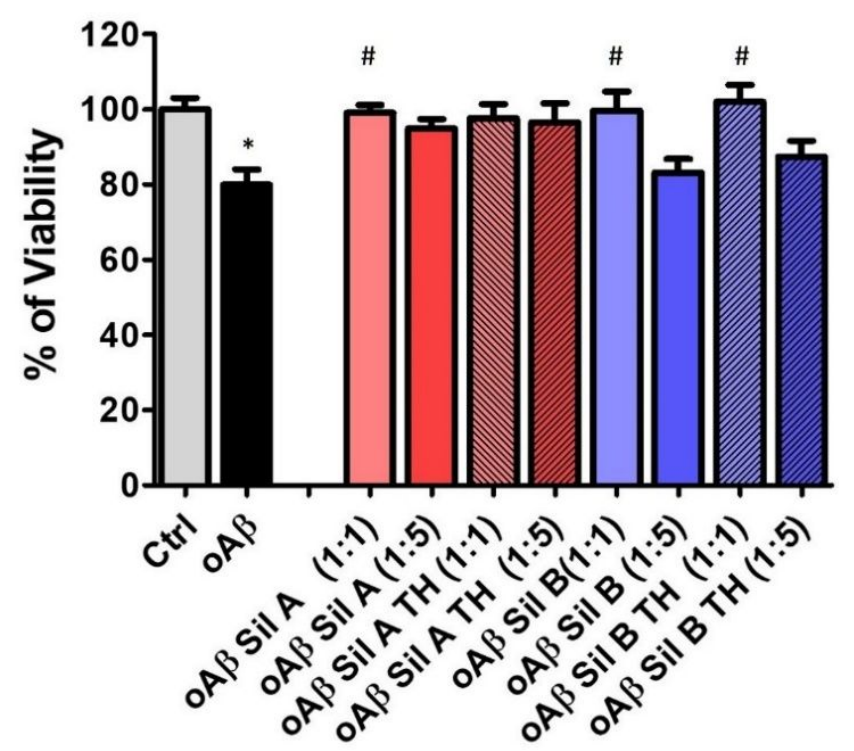

Figure S2. MTT assay of differentiated SH-SY5Y treated with preformed A $\beta$ oligomers for three hours, then exposed to conjugated/unconjugated Silybins for $48 \mathrm{hs}$. Bars are means \pm SEM of three independent experiments with $n=3 . \quad{ }^{*} P<0.05$ vs Ctrl by one-way ANOVA +Tukey's test. \# $<0.05$ vs o $A \beta$ by one-way ANOVA +Tukey's test. 

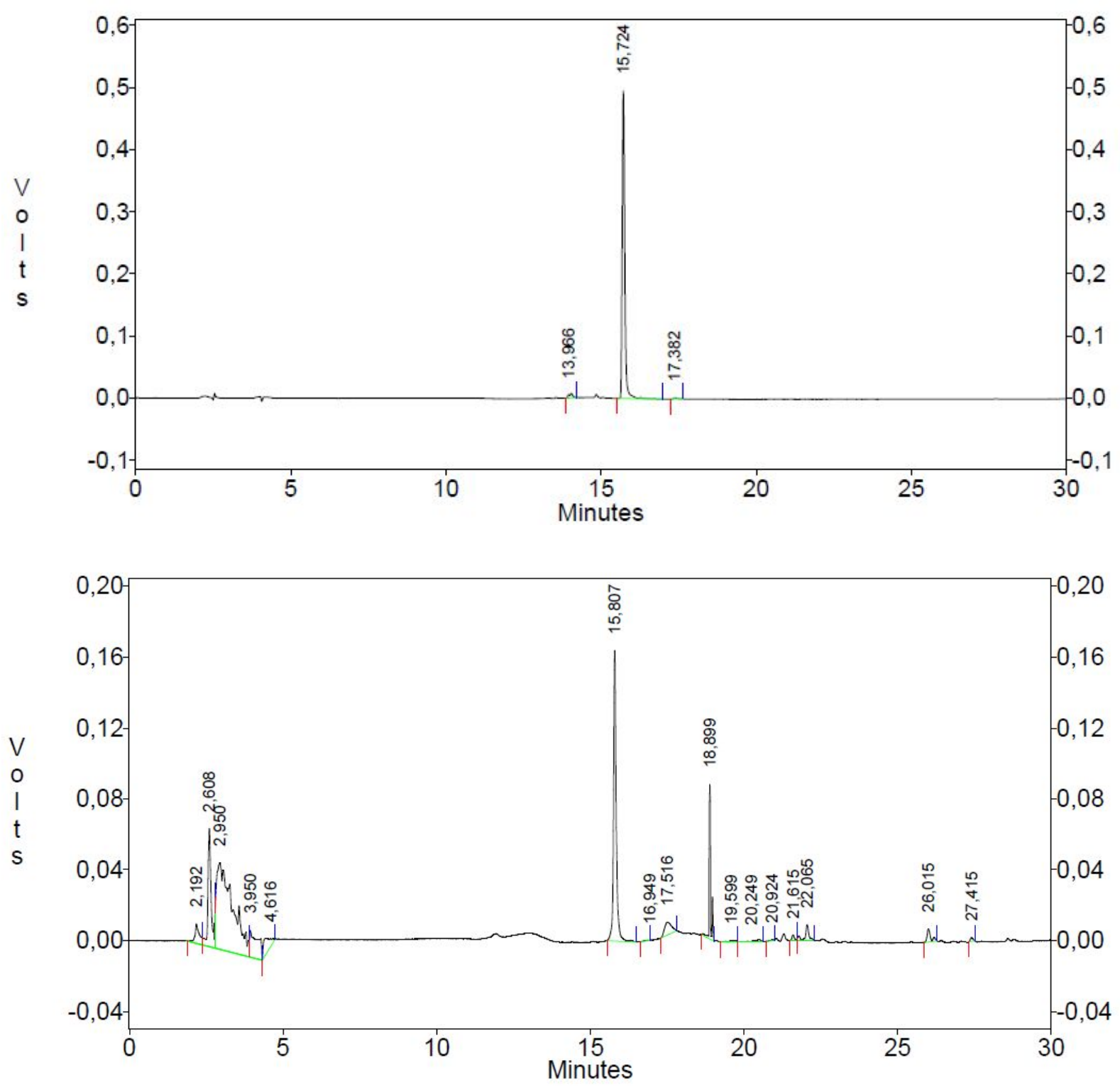

Figure S3. HPLC profiles of Sil A-p-TH in serum at $t=0 \min (A)$ and $t=24 h(B)$, as monitored by UV absorbance at $288 \mathrm{~nm}$. The analytes were eluted with $50 \mathrm{mM}$ ammonium acetate $(\mathrm{pH}=7.4)$ and acetonitrile (ACN) using the following gradient scheme: $5 \%$ ACN for $5 \mathrm{~min}$, and then $5-100 \%$ ACN over 30 $\min$ (flow rate $=1.0 \mathrm{~mL} / \mathrm{min}$ ). 

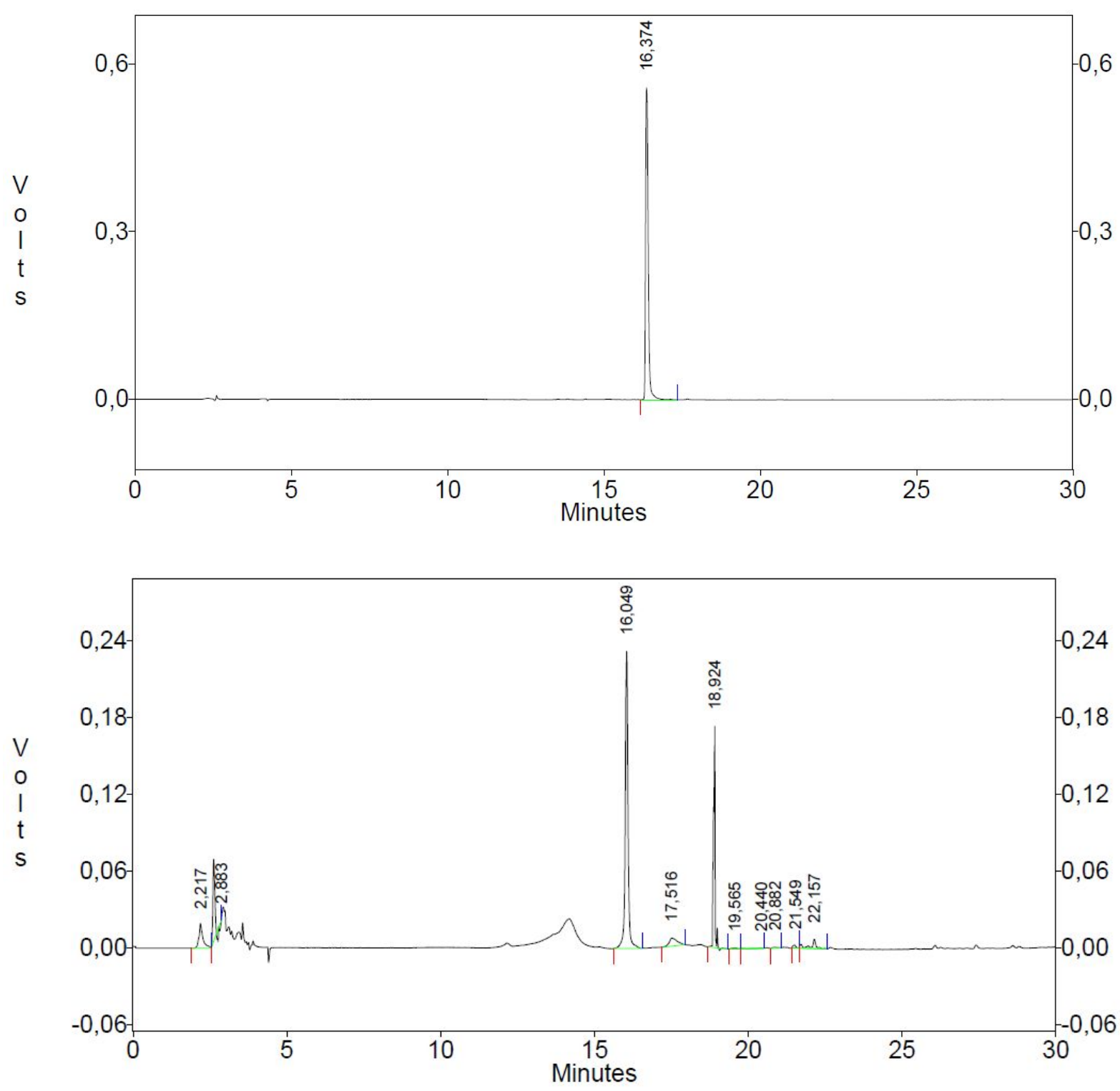

Figure S4. HPLC profiles of Sil B-p-TH in serum at $t=0 \min (A)$ and $t=24 h(B)$, as monitored by UV absorbance at $288 \mathrm{~nm}$. The analytes were eluted with $50 \mathrm{mM}$ ammonium acetate $(\mathrm{pH}=7.4)$ and acetonitrile (ACN) using the following gradient scheme: $5 \%$ ACN for $5 \mathrm{~min}$, and then $5-100 \%$ ACN over 30 $\min$ (flow rate $=1.0 \mathrm{~mL} / \mathrm{min}$ ). 


\section{References}

(1) Adzhubei A.A., Sternberg M.J.E., Makarov A.A. (2013) Polyproline-II Helix in Proteins: Structure and Function. J. Mol. Biol. 425 (12), 2100-2132. 